\title{
A high-resolution transmission-type x-ray spectrometer designed for observation of the $K \alpha$ transitions of highly charged high-Z ions
}

\author{
K. Widmann, ${ }^{\text {a) }}$ P. Beiersdorfer, G. V. Brown, J. R. Crespo López Urrutia, and V. Decaux \\ Department of Physics and Space Technology, Lawrence Livermore National Laboratory, Livermore, \\ California 94551 \\ D. W. Savin \\ Space Science Laboratory, University of California, Berkeley, California 94720
}

(Presented on 16 May 1996)

High-resolution reflection-type crystal spectrometers have been used for x-ray energies up to 13 $\mathrm{keV}$, e.g., the $K$-shell radiation of heliumlike $\mathrm{Kr}$. In order to extend crystal spectrometer measurements to higher energy $\mathrm{x}$ rays from higher- $Z$ elements, we employ the crystal in transmission. The geometry we use is known as DuMond geometry. Using such a transmission-type crystal x-ray spectrometer, we have measured the $K$-shell radiation of various highly charged high- $Z$ ions. In particular, we present a measurement of the $1 s 2 p^{1} P_{1} \rightarrow 1 s^{2}{ }^{1} S_{0}$ transition in heliumlike xenon, $\mathrm{Xe}^{52+}$. For this transition, we measure a linewidth of $34 \mathrm{eV}$, which demonstrates that the resolving power we achieved with the new spectrometer is on the order of 1000. (C) 1997 American Institute of Physics. [S0034-6748(97)68001-6]

\section{INTRODUCTION}

Spectroscopic measurements of the $\mathrm{x}$-ray radiation of plasmas, e.g., tokamak plasmas, provide rich and valuable information about various plasma parameters including the electron and ion temperatures, charge distribution, and bulk plasma motion. For those measurements, a high resolving power of the spectrometer is of utmost importance. Being able to resolve single transitions is the key to establishing a reliable and comprehensive atomic database including excitation, recombination, and ionization cross sections, as well as transition energies. With such an atomic database, x-ray spectroscopy can be applied successfully as a precise tool for plasma diagnostic. For very high-temperature plasmas spectroscopic diagnostics also require atomic data of highly charged ions. At our electron beam ion trap (EBIT) facility, highly charged ions are produced by means of a monoenergetic electron beam ${ }^{1,2}$ at energies up to and exceeding 200 $\mathrm{keV}$. A strong magnetic field, an electrostatic potential well along the beam axis, and the electron beam itself confine the highly charged ions, and make EBIT an ideal source for spectroscopic measurements of highly charged ions.

We have made high-resolution measurements for x-ray energies up to $13 \mathrm{keV}$, e.g., the $K$-shell radiation of heliumlike krypton, employing a reflection type crystal spectrometer. ${ }^{3}$ In order to extend crystal spectrometer measurements to higher energy $\mathrm{x}$ rays from higher- $Z$ elements, we have designed a transmission-type crystal spectrometer. In the following, we describe the design and implementation of this spectrometer on SuperEBIT, and present a measurement of the $1 s 2 p{ }^{1} P_{1} \rightarrow 1 s^{2}{ }^{1} S_{0}$ resonance line in heliumlike xenon, $\mathrm{Xe}^{52+}$. This is the first time that the $n=2 \rightarrow 1$ transitions of heliumlike and hydrogenlike xenon, $\mathrm{Xe}^{52+}$ and $\mathrm{Xe}^{53+}$, have been measured with such a high resolution. Precision measurements of heliumlike and hydrogenlike $K \alpha$

a) Also at Institut für Experimentalphysik, Technische Universität Graz, A-8010 Austria. transitions have been performed by Briand et al. ${ }^{4}$ using a germanium detector with a resolution of $270 \mathrm{eV}$. The current setup improves the spectral resolution by almost an order of magnitude.

\section{DESIGN AND IMPLEMENTATION}

Our transmission-type spectrometer design is based on the DuMond geometry ${ }^{5}$ employing a cylindrically bent crystal. The axes of this cylinder is parallel to the reflecting lattice planes which for the purpose of transmission-type spectrometry have to be perpendicular to the surface. The DuMond geometry requires that the radius of curvature of the crystal is the diameter of the so-called Rowland circle (see Fig. 1). SuperEBIT is a line source, $2 \mathrm{~cm}$ long and 60 $\mu \mathrm{m}$ wide. An aperture in the liquid helium shield constrains the accessible height of this line source to $1.2 \mathrm{~cm}$. Placing it on the Rowland circle strongly reduces the bandwidth of the diffracted $\mathrm{x}$ rays with the advantage that the throughput is tremendously increased at a given wavelength of interest. If the opening angle of the crystal is small enough, i.e., the distance between the $\mathrm{x}$-ray source and the crystal is much bigger than the length and height of the crystal, the diffracted $\mathrm{x}$ rays are quasimonochromatic. Using the spectrometer as a monochromator has the advantage that no position sensitive detector is necessary. Thus, solid state detectors, such as high-purity germanium detectors, can be used with almost $100 \%$ counting efficiency for $30 \mathrm{keV}$ photons. In addition, the energy dispersion of the germanium detector can be utilized for suppressing the background. For our measurement, we used a planar high-purity germanium detector. The dimensions of the germanium crystal are $16 \mathrm{~mm}$ in diameter and $10 \mathrm{~mm}$ in length. To obtain a spectrum, we rotate the crystal using a stepper motor mounted on a modified rotation stage. At each crystal position, we count the number of photons reaching the detector and tag this number with the current position of the stepper motor. We normalized each step 


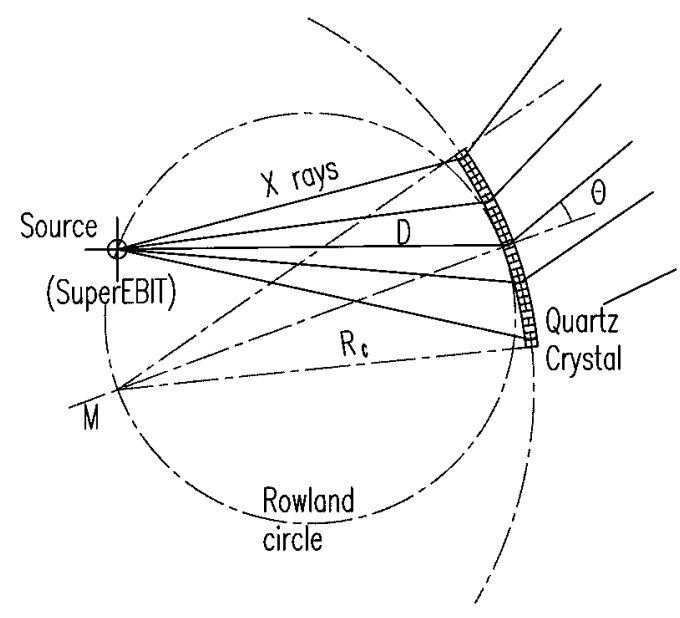

FIG. 1. Geometric requirements for a transmission-type crystal spectrometer using the DuMond setup. $\theta$ : Bragg angle; $D$ : distance source to crystal; $M$ : center of cylinder defined by the cylindrically bent crystal; $R_{c}$ : radius of curvature.

using the countrate of directly excited lines, emitted by the highly charged ions in the trap, which were measured with a second germanium detector.

A layout of the transmission-type crystal spectrometer for SuperEBIT is shown in Fig. 2. The spectrometer was set up to observe $K \alpha$ radiation of heliumlike and hydrogenlike xenon, $\mathrm{Xe}^{52+}$ and $\mathrm{Xe}^{53+}$, which are situated near $31 \mathrm{keV}$. We use a Quartz crystal cut perpendicular to the $(13 \overline{4} 0)$ planes, which has a $2 d$ spacing of $2 d=2.3604 \AA$. Thus, the nominal Bragg angle is around $\theta=9.9^{\circ}$. The radius of curvature of the crystal was $R_{c}=(2713.8 \pm 3.2) \mathrm{mm}$ and was measured using an optical setup. The illuminated area of the crystal is (60 $\times 40) \mathrm{mm}^{2}$.

Positioning the crystal so that the electron beam is part of the Rowland circle is nontrivial. Perfect alignment is achieved when

$$
D=R_{c} \sin \theta
$$

$D$ being the distance between the source and the crystal, $R_{c}$ the radius of curvature of the crystal, and $\theta$ the Bragg angle (see Fig. 1). Refering to Eq. (1) the required distance between the source and the crystal must be $D_{\text {theor }}=(2673.6 \pm 3.2) \mathrm{mm}$ for our crystal. The distance be-

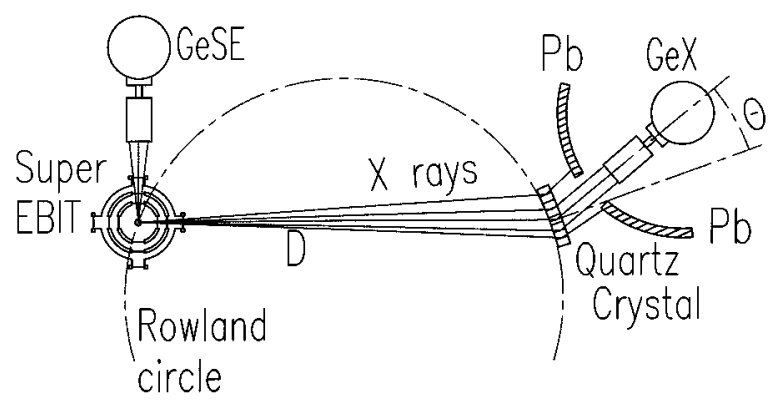

FIG. 2. Layout of the transmission-type crystal spectrometer in the horizontal plane of SuperEBIT. The electron beam is perpendicular to the page. GeSE and GeX are high-purity germanium detectors; $\theta$ : Bragg angle; $D$ : distance source to crystal; $\mathrm{Pb}$ : lead shielding.

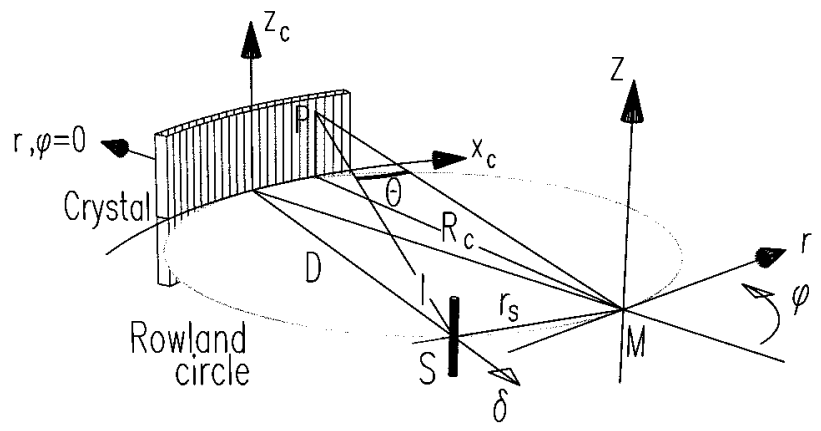

FIG. 3. Sketch to visualize the system of coordinates and the meaning of the parameters used to calculate the Bragg angle at any point on the crystal and for any location of the source. Labels are described in the text, see Eq. (2).

tween the crystal and the center of SuperEBIT was measured to be $D=(2675 \pm 5) \mathrm{mm}$. Therefore, the "misalignment" of the crystal could be up to $10 \mathrm{~mm}$ too close to the source, i.e., $\delta=-10 \mathrm{~mm}$, or up to $7 \mathrm{~mm}$ too far away from the source, $\delta=+7 \mathrm{~mm}$.

Even under the assumption that the crystal is perfectly aligned, the $\mathrm{x}$ rays diffracted in transmission still have a certain energy spread, i.e., they are only quasimonochromatic. By gauging the difference in the angle across the crystal that meet the Bragg condition, we calculated the energy spread of the diffracted $\mathrm{x}$-ray photons reaching the detector. These calculations help to estimate how accurate the crystal has to be aligned in respect to the electron beam so that the alignment is not the limiting factor for the resolving power of the spectrometer.

For this calculation, we introduce a system of polar coordinates $(r, \varphi, z)$, illustrated in Fig. 3. The plane going through the middle of the trap and the crystal is the $z=0$ plane. The center, $M$, of the circle determining the curvature of the crystal is the origin $(0,0,0)$ of the system of coordinates. For any point $P\left(R_{c}, \varphi, z\right)$ on the crystal, the Bragg angle with respect to the $\mathrm{x}$ rays coming from the source $S\left(r_{s}, \varphi_{s}, 0\right)$ can be extracted from the following expression:

$$
\cos \theta=\frac{r^{2}-R_{c} r_{s} \cos \left(\varphi_{s}-\varphi\right)}{r l} .
$$

$R_{c}$ is the radius of curvature of the crystal, $r$ is the distance between the point $P$ and the origin $M, r_{s}$ is the distance between the source $S$ and $M, \varphi$ and $\varphi_{s}$ are the angular coordinates of $P$ and $S$, respectively, and $l$ is the distance between $P$ and $S$. The energy can be calculated using the Bragg condition

$$
E=\frac{h c n}{2 d \sin \theta},
$$

where $h$ is the Planck constant, $c$ the speed of light, $h c$ $=12398.42 \mathrm{eV} \cdot \AA, n$ is the order of diffraction, $d$ is the lattice spacing, and $\theta$ the Bragg angle. Figure 4 shows the result of this calculation displaying the energy spread across the illuminated part of the crystal. The detector area projected onto the crystal plane $\left(x_{c}, z_{c}\right)$, using a ray tracing method, covers the horizontal region of $x_{c}< \pm 7.5 \mathrm{~mm}$ and the vertical region of $z_{c}< \pm 13.5 \mathrm{~mm}$, taking into account the accessible height of the trap. The misalignment of the source $S$, i.e., the 

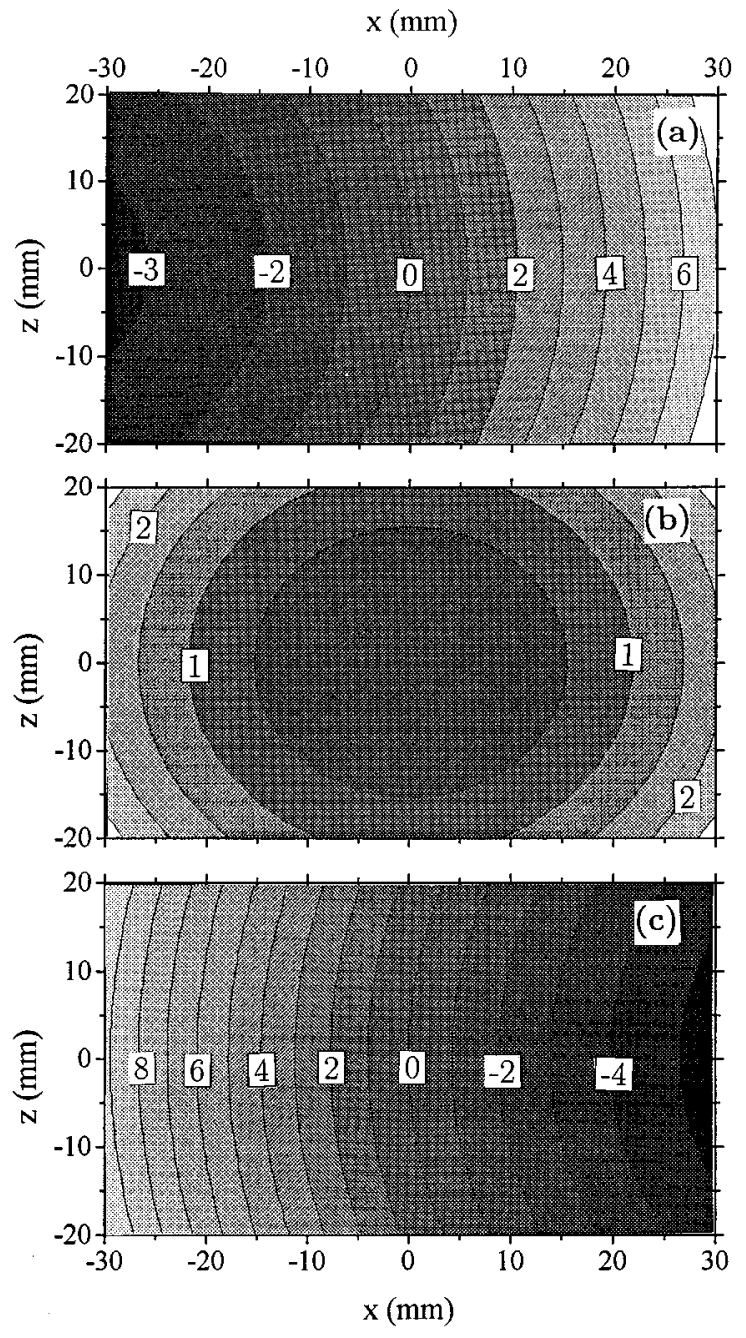

FIG. 4. Energy difference (in eV) between the transition energy of interest, in this case $30630 \mathrm{eV}$, and the energies of the diffracted $\mathrm{x}$ rays across the illuminated area of the crystal. X-ray photons which are diffracted in the area $x_{c} \epsilon[-7.5 ; 7.5] \mathrm{mm}$ and $z_{c} \epsilon[-13.5 ; 13.5] \mathrm{mm}$ are reaching the detector. (a) Crystal is $7 \mathrm{~mm}$ too far away from SuperEBIT, $\delta=7 \mathrm{~mm}$. (b) Exact alignment of the crystal, $\delta=0 \mathrm{~mm}$. (c) Crystal is $10 \mathrm{~mm}$ too close to $\mathrm{Su}-$ perEBIT, $\delta=-10 \mathrm{~mm}$.

distance between the source and the Rowland circle, is expressed in the length $\delta$. Negative values of $\delta$ describe a shift of the source towards the crystal, i.e., inside the Rowland circle, positive numbers of $\delta$ indicate a shift away from the crystal. At perfect alignment, i.e., $\delta=0 \mathrm{~mm}$, the bandwidth of the $\mathrm{x}$ rays diffracted in transmission is $\Delta E_{c}=2.7 \mathrm{eV}$ for the whole crystal and less than $\Delta E_{\text {det }}<0.5 \mathrm{eV}$ across the detector area [see Fig. 4(b)]. As mentioned above, the misalignment in our setup can be between $\delta=+7$ and $-10 \mathrm{~mm}$. The first case, $\delta=+7 \mathrm{~mm}$, yields a bandwidth of $\Delta E_{c} \approx 10 \mathrm{eV}$ and $\Delta E_{\mathrm{det}} \approx 2 \mathrm{eV}$. For a shift of $\delta= \pm 10 \mathrm{~mm}$, the energy spread of the diffracted $\mathrm{x}$ rays is $\Delta E_{c} \approx 16 \mathrm{eV}$ and $\Delta E_{\mathrm{det}} \approx 3.5 \mathrm{eV}$.

The influence of the finite width of the source $\Delta x$ on the resolution of the transmission-type spectrometer is given by

$$
\Delta \theta=\arctan \left(\frac{\Delta x}{D}\right) .
$$

$\Delta \theta$ is the Bragg angle interval "seen" by the crystal due to the lateral extension of the source, and $D$ is the distance between the source and the crystal. With a beam width of $\Delta x_{\text {beam }}=60 \mu \mathrm{m}$ we derive, using Eq. (4), a maximal difference of $\Delta \theta=0.0013^{\circ}$ in the Bragg angle. This gives an energy spread of about $4 \mathrm{eV}$ for each point of the crystal, i.e., for every diffracted x-ray photon. Therefore, the nominal resolution of our transmission-type spectrometer cannot be better than $\Delta E=4.5 \mathrm{eV}$, and the resolving power not better than $E / \Delta E=6800$.

Equation (4) can be applied for the discussion of the limitation of the resolving power due to the quality of the focus of the bent crystal. For that purpose, $\Delta x$ has to be the width of the focus. The optical setup that was used to measure the radius of curvature of the crystal only allows an estimate of the upper limit of the focal width $\Delta x_{\text {focus }}$. Our estimate for this crystal is $\Delta x_{\text {focus }}<0.5 \mathrm{~mm}$, which gives an upper limit for the band width of $\Delta E \approx 33 \mathrm{eV}$. The effects causing a broadening of the band width of the in transmission diffracted $\mathrm{x}$-ray photons add linearly. This means the resolution of our transmission-type crystal spectrometer is $\Delta E=40 \mathrm{eV}$ or better. Thus, the resolving power should be $E / \Delta E>750$.

\section{MEASUREMENTS}

Heliumlike and hydrogenlike xenon have ionization potentials of $E_{\text {ion }}=40.3 \mathrm{keV}$ and $E_{\text {ion }}=41.3 \mathrm{keV}$, respectively. In order to obtain a charge balance, which is dominated by heliumlike xenon, we set the electron beam energy to about $114 \mathrm{keV}$. The beam current was $270 \mathrm{~mA}$. The space charge of the electron beam creates a radial potential which for these operating parameters is about $14 \mathrm{~V}$, and the axial trap potential was set to $10 \mathrm{~V}$. For the observation of the xenon spectra neutral xenon atoms are injected into SuperEBIT by means of a gas injector.

The spectrum of the direct excitation lines and the radiative recombination lines, taken with the germanium detector labeled GeSE in Fig. 2 is shown in Fig. 5. Such spectra were taken continuously throughout the whole experiment to monitor the charge balance. Additionally, the counts of the $K \alpha$ transitions were taken to normalize the stepping time, e.g., after collecting 12000 counts in the $K \alpha$ energy interval the crystal was turned one step. The change in the Bragg angle was $0.000741^{\circ} \pm 0.000001^{\circ}$ per step which is equivalent to an energy change of $2.2 \mathrm{eV}$ for the diffracted photons at these x-ray energies, i.e., around $30600 \mathrm{eV}$. At each step, we counted the $x$-ray photons reaching the second germanium detector, labeled GeX in Fig. 2. Suppressing all x-ray photons which did not fall into the xenon $K \alpha$ energy interval was necessary to lower the background.

Figure 6 presents the result of 69 scans over the same spectral region. The spectrum features the $1 s 2 p^{1} P_{1} \rightarrow 1 s^{2}{ }^{1} S_{0}$ transition of heliumlike xenon, $\mathrm{Xe}^{52+}$. It took $116 \mathrm{~h}$ to collect the amount of counts shown in Fig. 6. The line contains about 110 counts, which gives an overall countrate of 1 count per hour for the $1 s 2 p{ }^{1} P_{1} \rightarrow 1 s^{2}{ }^{1} S_{0}$ photons. For the background, a countrate of 3 counts per hour can be derived. However, the signal to noise ratio is much better than $\mathrm{S} / \mathrm{N}=1 / 3$ because the data are collected, literally, one step at a time. Each channel represents, actu- 

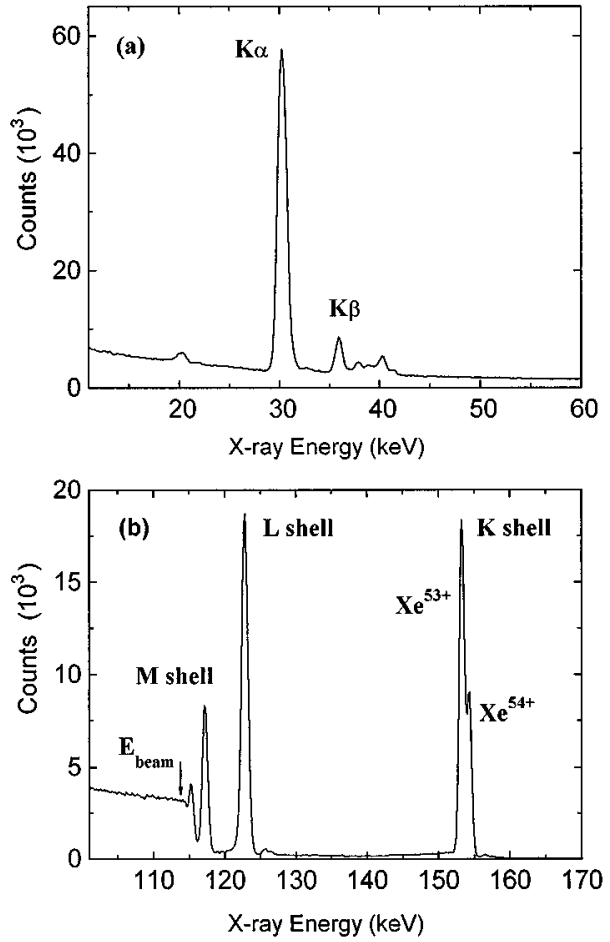

FIG. 5. Spectra taken with the germanium detector (GeSE in Fig. 2). The beam energy was set to $114 \mathrm{keV}$, the beam current was $270 \mathrm{~mA}$. (a) Direct excitation lines of mostly heliumlike $\mathrm{Xe}^{52+}$. The stepping time of the stepper motor rotating the crystal was linked to the countrate of the $K \alpha$ peak. (b) Spectrum of the radiative recombination (RR) lines of xenon. The intensity ratio between the $K$ - and $L$-shell RR lines was used to monitor the charge balance in the trap.

ally, only $3 \mathrm{~h}$ worth of data. Calculating the countrate per channel, we get 3 counts per hour and per channel for the background and almost 7 counts per hour and per channel for the $1 s 2 p{ }^{1} P_{1} \rightarrow 1 s^{2}{ }^{1} S_{0}$ photons, when scanning over the line. Therefore, $\mathrm{S} / \mathrm{N} \approx 2 / 1$, which is well represented in the spectrum.

In Fig. 6, one channel represents the sum of the counts obtained during three consecutive steps. The dispersion is 6.6 $\mathrm{eV}$ per channel. Using a simple Gaussian fit, we obtain a full width at half maximum of $(34 \pm 5) \mathrm{eV}$. Therefore, the measured resolving power of our transmission-type crystal spectrometer is about 900 . This shows that the main limitation on resolving power is due to the poor focusing ability of the

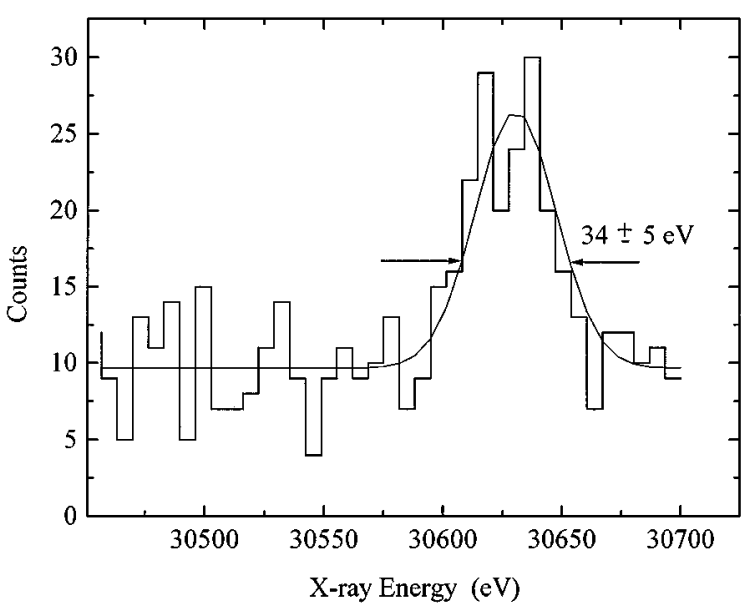

FIG. 6. First highly resolved spectrum of the $1 s 2 p{ }^{1} P_{1} \rightarrow 1 s^{2}{ }^{1} S_{0}$ transition of He-like $\mathrm{Xe}^{52+}$ demonstrating the successful implementation of the transmission-type crystal spectrometer at SuperEBIT. For x-ray energies around $31 \mathrm{keV}$ the resolving power of this spectrometer is around 900.

crystal, as discussed above. Using the measured value of the resolution, i.e., $(34 \pm 5) \mathrm{eV}$, the focal width of the crystal can be determined: $\Delta x_{\text {focus }}=(0.43 \pm 0.10) \mathrm{mm}$, which is somewhat smaller than the upper limit determined by our optical focusing measurement.

Further improvement of the resolution depends on the ability of bending the crystal with a higher accuracy, yielding a smaller focal width.

\section{ACKNOWLEDGMENTS}

The authors thank E. Magee and D. Nelson for their technical support. This work was performed under the auspices of U.S. DOE by Lawrence Livermore National Laboratory under Contract No. W-7405-ENG-48.

${ }^{1}$ M. A. Levine, R. E. Marrs, J. R. Henderson, D. A. Knapp, and M. B. Schneider, Phys. Scr. T 22, 157 (1988).

${ }^{2}$ P. Beiersdorier, R. Cauble, S. Chantrenne, M. Chen, N. DelGrande, D. Knapp, R. Marrs, A. Osterheld, K. Reed, M. Schneider, J. Scofield, B. Wargelin, K. Wong, D. Vogel, and R. Zasadzinski, in Proceedings from the Tenth International Colloquium on UV and X-Ray Spectroscopy of Astrophysical and Laboratory Plasmas, edited by E. Silver and S. Kahn (Cambridge University Press, Cambridge, 1993), p. 59.

${ }^{3}$ K. Widmann, P. Beiersdorfer, and V. Decaux, Phys. Rev. A 53, 2200 (1996).

${ }^{4}$ J. P. Briand, P. Indelicato, A. Simionovici, V. San Vicente, D. Liesen, and D. Dietrich, Europhys. Lett. 9, 225 (1989).

${ }^{5}$ J. W. M. DuMond, Rev. Sci. Instrum. 18, 626 (1947). 